\title{
Matrix Geometry and Coherent States
}

\author{
Goro Ishiki* \\ Graduate School of Pure and Applied Science, \\ University of Tsukuba, Tsukuba, Ibaraki 305-8571, Japan \\ Center for Integrated Research in Fundamental Science and Engineering (CiRfSE), \\ University of Tsukuba, Tsukuba, Ibaraki 305-8571, Japan \\ E-mail: ishiki@het.ph.tsukuba.ac.jp
}

We consider the commutative limit of matrix geometry described by some Hermitian matrices. We propose a prescription to define the commutative space and its tangent space in terms of given matrix configurations. We also show that a certain class of matrix geometry possesses Poisson structures in the commutative limit. We find a relation between these geometric objects and matrix configurations which define the matrix geometry.

Proceedings of the Corfu Summer Institute 2015 "School and Workshops on Elementary Particle Physics and Gravity"

1-27 September 2015

Corfu, Greece

${ }^{*}$ Speaker. 


\section{Introduction}

In the matrix model formulations of the string theory and M-theory [1,2], configurations of fundamental objects such as strings and membranes are described in terms of some Hermitian matrices. In order to gain a deeper understanding of such formulations, one would need to clarify a relation between the matrix configurations and the usual differential geometric description of strings and membranes.

In particular, the case in which strings or membranes are embedded in the flat target space is relevant to the matrix model formulations. The matrix geometry for an embedded space in the flat space $R^{D}$ can be defined in terms of $D$ Hermitian matrices $X^{\mu}(\mu=1,2, \cdots, D)$, which can be regarded as quantized form of the embedding function of objects in string theory such as strings or D-branes. For the matrices with a fixed size $N$, one can associate a commutative space $\mathscr{M}$ for the given matrices by using the method proposed in [3] (see also [4, 5]). Here, $\mathscr{M}$ is defined by a set of loci of zero eigenmodes of the matrix Dirac operator, $D(y)=\gamma_{\mu} \otimes\left(X^{\mu}-y^{\mu}\right)$, where $y^{\mu}$ are real parameters. In the string theory, if $X^{\mu}$ represent a certain configuration of bosons on $N$ coincident D0-branes, $D(y)$ appears just as the Dirac operator in the low-energy effective action of open strings connecting the D0-branes with another probe D0-brane at the position $y$. The zero eigenmodes are just massless modes of open strings. Thus at $y \in \mathscr{M}$, the probe brane feels the presence of one of the other D0-branes. So the loci of zero eigenstates correspond to the shape of a D-brane formed by the D0-branes.

In this paper, we consider a large- $N$ family of $X^{\mu}$. Namely, we assume that $\left\{X_{\mu}^{(N)} \mid N \in I\right\}$ is given, where $X_{\mu}^{(N)}(\mu=1, \cdots, D)$ are $N \times N$ Hermitian matrices and $I$ is a fixed index set made of a strictly increasing infinite sequence of natural numbers. In particular, we consider matrices which satisfy

$$
\left[X^{\mu}, X^{v}\right]=\frac{i}{C_{N}} W^{\mu v}(X)+\mathscr{O}\left(1 / C_{N}^{2}\right),
$$

where, $W^{\mu v}(X)$ is a polynomial of $X^{\mu}$ such that its degree and coefficients do not depend on $N$. $C_{N}$ is a real $N$-dependent constant such that $C_{N} \rightarrow \infty$ as $N \rightarrow \infty$. So the large- $N$ limit in this setup corresponds to the commutative limit. We also call it classical limit since this corresponds to the classical limit in quantum mechanics, where $X^{1}$ and $X^{2}$ correspond to the canonical operators, $\hat{p}$ and $\hat{q}$.

In this paper, we explain how to define the classical (commutative) space and its tangent space in terms of the given matrices. We also show that if the matrices $X^{\mu}$ satisfy (1.1), the associated classical space possesses a Poisson structure. We will find relations between the given matrix configuration and the geometric objects, such as the Poisson structure and a projection operator onto the tangent space. These results were firstly presented in the publication [6].

The condition 1.11 restricts possible configurations of the matrices. However, this condition is satisfied by most known examples of fuzzy spaces, such as fuzzy sphere, fuzzy torus and fuzzy $C P^{n}$. The condition is also satisfied by the nontrivial one-parameter family of noncommutative spaces found in [7]. Furthermore, for any matrices $X^{\mu}$ satisfying [1.1), one can add a perturbation given by polynomials of $X^{\mu}$ as $X^{\mu} \rightarrow X^{\mu}+f^{\mu}(X)$. If the definitions of $f^{\mu}(x)$ are independent of $N$, the condition (1.1) is also satisfied by the perturbed configuration up to any order of the 
perturbation. Thus, our set up is also relevant to matrix models expanded around some background configurations.

The motivation to consider the large- $N$ sequence, not just a fixed matrix size, partially lies in the matrix regularization [8]. The matrix regularization is a sort of approximation of functions on a symplectic manifold $\mathscr{M}$ using the matrix algebra $M_{N}(C)$. The matrix regularization is defined in terms of a sequence $\left\{T_{N}\right\}$ of linear maps $T_{N}: C(\mathscr{M}) \rightarrow M_{N}(C)$ such that the ring structure is preserved and the Poisson bracket is mapped to the commutator of matrices in the large- $N$ limit. Namely, $\left\|T_{N}(f) T_{N}(g)-T_{N}(f g)\right\| \rightarrow 0$ and $\left\|T_{N}(\{f, g\})-i N\left[T_{N}(f), T_{N}(g)\right]\right\| \rightarrow 0$, where $\|\cdot\|$ is a matrix norm. If we regard $X^{\mu}$ as images of the embedding function under $T_{N}$, a problem of finding the commutative geometry from given matrices is just the inverse problem of the construction of matrix regularization. Understanding the inverse problem will shed light on the construction of the matrix regularization.

This paper is organized as follows. In section 2 and 3 we define the classical space and its tangent space in terms of the given matrices, respectively. In section 4 we show that the classical space possesses a Poisson structure if 1.1 is satisfied. In section 5, we consider some examples for this construction. For fuzzy sphere and fuzzy torus, we show explicit forms of the geometric objects defined in the previous sections. Section 6 is devoted to a summary.

\section{Classical space}

In this section, we consider the classical limit of matrix geometry and define the classical space $\mathscr{M}$ in terms of the given matrices. We adopt the method proposed in [6].

We first define an $N \times N$ Hermitian matrix, which is called Hamiltonian, by

$$
H(y)=\frac{1}{2}\left(X^{\mu}-y^{\mu} \mathbf{1}\right)^{2} .
$$

where $y^{\mu}(\mu=1,2, \ldots, D)$ are real parameters. We introduce the eigenstates of the Hamiltonian as

$$
H(y)|n, y\rangle=E_{n}(y)|n, y\rangle, \quad(n=0, \cdots, N-1)
$$

where we assume $E_{i} \leq E_{i+1}$ for any $i$ and the states are normalized as $\langle n, y \mid m, y\rangle=\delta_{m n}$. Then, we define the classical spaces as loci in $R^{D}$ of zeros of the ground state energy $E_{0}(y)$ of the Hamiltonian in the commutative limit $N \rightarrow \infty$. Namely, we write

$$
\mathscr{M}=\left\{y \in R^{D} \mid f(y)=0\right\},
$$

where $f(y)$ is the large- $N$ limit of the ground state energy,

$$
f(y)=\lim _{N \rightarrow \infty} E_{0}(y) .
$$

This definition allows an intuitive interpretation. The ground state energy can be written as

$$
E_{0}(y)=\frac{1}{2}\left(\Delta X^{\mu}\right)^{2}+\frac{1}{2}\left(\left\langle X^{\mu}\right\rangle-y^{\mu}\right)^{2}
$$

where $\Delta X^{\mu}$ and $\left\langle X^{\mu}\right\rangle$ are the standard deviation and expectation value of $X^{\mu}$ with respect to the ground state, respectively. When the ground state energy goes to zero, both $\Delta X^{\mu}$ and $\left\langle X^{\mu}\right\rangle-y^{\mu}$ 
go to zero. Thus, if the ground state energy vanishes, there exists a wave function such that its wave packet shrinks to the point $y$. Conversely, suppose that there exists a wave packet such that both of the standard deviation and the expectation value of $X^{\mu}$ minus $y^{\mu}$ are vanishing for the corresponding state. Then, the expectation value of the Hamiltonian for that state is also vanishing. Since the ground state energy is the smallest expectation value among all possible states, it is also vanishing. Therefore, (2.3) is the space on which there exist shrinking wave packets, which can naturally be identified with points in the classical limit. Those wave functions are analogues of the coherent states in quantum mechanics ${ }^{1}$.

Note that in principle the function $f(y)$ can be computed from the given matrices. Thus, the definition (2.3) provides a practical way of finding the classical space form the given matrix configurations.

However, for a general (random) sequence of matrices, the classical space 2.3) is the most likely to be an empty set. A necessary condition to have a non-empty set is that the matrices $X^{\mu}$ become commuting with each other in the large- $N$ limit. This is because the ground state energy $E_{0}(y)$ is bounded from below by $\left|\left\langle\left[X^{\mu}, X^{v}\right]\right\rangle\right| / 2$ for arbitrary $\mu$ and $v$ (We can derive this relation in exactly the same way as the standard derivation of the uncertainty inequality in quantum mechanics.). Since we consider (1.1) in this paper, this necessary condition is always satisfied.

The relation (1.1) also allows us to relate the definition of the classical space with that in [3]. In [3], two-dimensional surfaces embedded in $R^{3}$ are considered (See also [12] for applications to various configurations of surfaces and [13] for higher dimensional extensions.). Here, the classical space is defined for any fixed $N$ as the loci of zero eigenmodes of the Dirac operator $D(y)=$ $\sigma_{i} \otimes\left(X^{i}-y^{i}\right)$, where $\sigma_{i}$ are the Pauli matrices. Note that $D^{2}(y)$ is equal to our Hamiltonian plus terms proportional to commutators $\left[X^{\mu}, X^{v}\right]$. Under the condition 11.1), the commutators become negligible in the large- $N$ limit. Therefore, in the large- $N$ limit, the classical space defined by the Hamiltonian is the same as the space defined by the Dirac operator.

In this paper, we assume that the classical space is a smooth manifold, Precisely speaking, we assume that $\mathscr{M}$ is a non-empty connected subset of $R^{D}$ and there exists a neighbourhood of $\mathscr{M}$ on which the function $f(y)$ is smooth. As we will see in the following, the latter condition is required for $\mathscr{M}$ to be a smooth manifold and to have a well defined tangent space.

\section{Tangent space}

In order to consider differential geometric objects on $\mathscr{M}$, we then define the tangent space of $\mathscr{M}$ using the given matrices. For each $y \in \mathscr{M}$, let us consider an arbitrary $D$-dimensional vector $B^{\mu}(y)$. There should be a projection operator, which projects $B^{\mu}$ onto the tangent components along $\mathscr{M}$. As we will show below, the projection operator is given by [6]

$$
P^{\mu}{ }_{v}(y)=\delta_{v}^{\mu}-\partial^{\mu} \partial_{v} f(y)
$$

Here and thereafter, we raise and lower the indices $\mu, v$ by the Kronecker delta, which is just the flat metric on the target space. So we just have $\partial^{\mu}=\partial_{\mu}$. In terms of the projection operator, the

\footnotetext{
${ }^{1}$ See [9-11] for applications of coherent states in describing matrix geometries.
} 
tangent vectors are defined as those satisfying

$$
P^{\mu}{ }_{v}(y) B^{v}(y)=B^{\mu}(y)
$$

for $y \in \mathscr{M}$.

The projection operator is positive definite on the tangent vectors and it gives a metric on $\mathscr{M}$. This corresponds to the induced metric of the embedding $\mathscr{M} \rightarrow R^{D}$.

We can also relate the projection $P^{\mu}{ }_{v}$ to the given matrices by using the perturbation theory of quantum mechanics. For a small change of the variables, $y^{\mu} \rightarrow y^{\mu}+\varepsilon^{\mu}$, the Hamiltonian varies by $\varepsilon \cdot(y-X)+\frac{1}{2} \varepsilon^{2}$. The perturbative corrections of the ground state energy is written in terms of the variation of the Hamiltonian through formulas in perturbation theory. This relates derivatives of the ground state energy with $X^{\mu}$. These derivatives in turn give derivatives of $f(y)$ in the large- $N$ limit, which are related to $P^{\mu}{ }_{v}$ by (3.1). Thus, this relation connects $P^{\mu}{ }_{v}$ to the given matrices. More specifically, $P^{\mu}{ }_{v}$ is explicitly written as

$$
P^{\mu}{ }_{v}(y)=2 \lim _{N \rightarrow \infty} \sum_{n \neq 0} \operatorname{Re} \frac{\left\langle 0, y\left|X^{\mu}\right| n, y\right\rangle\left\langle n, y\left|X_{v}\right| 0, y\right\rangle}{E_{n}(y)-E_{0}(y)} .
$$

This expression as well as 3.1 provides a practical way of computing $\left\{P^{\mu}{ }_{v}\right\}$ from the given matrices. Here, in order to write down (3.3), we have assumed that the ground state is non-degenerate on $\mathscr{M}$.

Below, we prove the projectivity of $\left\{P^{\mu}{ }_{v}\right\}$. We first derive some useful equations. For any normalized vector $|\psi\rangle$, we have

$$
\lim _{N \rightarrow \infty}\left\langle\psi\left|y^{\mu}-X^{\mu}\right| 0, y\right\rangle=0
$$

for $y \in \mathscr{M}$. This follows from the Cauchy-Schwarz inequality and the fact that $\lim _{N \rightarrow \infty} E_{0}(y)=0$ for $y \in \mathscr{M}$. This relation can be generalized to

$$
\lim _{N \rightarrow \infty}\langle\psi|h(y)-h(X)| 0, y\rangle=0, \quad(y \in \mathscr{M})
$$

where $h(y)$ is any polynomial with $N$-independent degree and coefficients. Here, we have assumed that $X_{\mu}$ are norm-bounded in the large- $N$ limit. This corresponds to the case where the classical space is a compact subspace of $R^{D}$.

Firstly, we write $P^{\mu}{ }_{v}(y)$ at $y \in \mathscr{M}$ as

$$
P^{\mu}{ }_{v}(y)=\delta_{v}^{\mu}-\lim _{N \rightarrow \infty} \partial_{v}\left\langle 0, y\left|y^{\mu}-X^{\mu}\right| 0, y\right\rangle .
$$

We note that $\left\langle 0, y\left|y^{\mu}-X^{\mu}\right| 0, y\right\rangle$ is vanishing for any $y \in \mathscr{M}$ in the large- $N$ limit because of 3.4p. Then its derivatives along tangent directions are also vanishing. Thus, for any tangent vector $\varepsilon_{\|}^{\mu}$ at $y \in \mathscr{M}$, we have

$$
P^{\mu}{ }_{v}(y) \varepsilon_{\|}^{v}=\varepsilon_{\|}^{\mu}
$$

This means that $P^{\mu}{ }_{v}$ is closed on tangent vectors and is equal to $\delta_{v}^{\mu}$. 
Secondly, we consider an arbitrary normal vector $\varepsilon_{\perp}^{\mu}$ at $y \in \mathscr{M}$. We will show that

$$
P^{\mu}{ }_{v}(y) \varepsilon_{\perp \mu} \varepsilon_{\perp}^{v}=0 .
$$

The equations (3.7) and (3.8) are equivalent to the projectivity of $P^{\mu}{ }_{v}(y)$. Note that the left-hand side of (3.8) appears in a power series expansion of $f\left(y+\varepsilon_{\perp}\right)$ in $\varepsilon_{\perp}$. Indeed, it is expanded as

$$
f\left(y+\varepsilon_{\perp}\right)=\frac{1}{2}\left(\delta_{v}^{\mu}-P^{\mu}{ }_{v}(y)\right) \varepsilon_{\perp \mu} \varepsilon_{\perp}^{v}+\cdots,
$$

for $y \in \mathscr{M}$. In the following, we evaluate $f\left(y+\varepsilon_{\perp}\right)$ and show that it is given as

$$
f\left(y+\varepsilon_{\perp}\right)=\frac{1}{2}\left|\varepsilon_{\perp}\right|^{2}+\mathscr{O}\left(\left|\varepsilon_{\perp}\right|^{3}\right) .
$$

The equation (3.8) follows from (3.9) and (3.10). So below, we focus on a proof of (3.10).

In order to prove (3.10), we first prove

$$
\lim _{N \rightarrow \infty} K_{N}\left\langle 0, y_{1}|H(y)| 0, y_{2}\right\rangle=\frac{1}{2}\left(y_{2}^{\mu}-y^{\mu}\right)^{2} \delta^{(D)}\left(y_{1}-y_{2}\right), \quad\left(y_{1}, y_{2} \in \mathscr{M}\right)
$$

where $K_{N}$ is a real positive constant defined below. We can compute the object on the left-hand side as

$$
\left\langle 0, y_{1}|H(y)| 0, y_{2}\right\rangle=\left(E_{0}\left(y_{2}\right)+\frac{1}{2}\left(y_{2}^{\mu}-y^{\mu}\right)^{2}\right)\left\langle 0, y_{1} \mid 0, y_{2}\right\rangle+\left(y_{2}^{\mu}-y^{\mu}\right)\left\langle 0, y_{1}\left|X_{\mu}-y_{2 \mu}\right| 0, y_{2}\right\rangle .
$$

Because of (3.4), the inner product $\left\langle 0, y_{1} \mid 0, y_{2}\right\rangle$ satisfies

$$
\lim _{N \rightarrow \infty}\left(y_{2}^{\mu}-y_{1}^{\mu}\right)\left\langle 0, y_{1} \mid 0, y_{2}\right\rangle=0 . \quad\left(y_{1}, y_{2} \in \mathscr{M}\right)
$$

This shows that the ground states at different points are orthogonal to each other in the large- $N$ limit. So they must satisfy

$$
\lim _{N \rightarrow \infty} K_{N}\left\langle 0, y_{1} \mid 0, y_{2}\right\rangle=\delta^{(D)}\left(y_{1}-y_{2}\right) . \quad\left(y_{1}, y_{2} \in \mathscr{M}\right)
$$

Here, we introduced the constant $K_{N}$ as a proportionality constant. By applying (3.4) and (3.14) to (3.12), we obtain 3.11.

Then, we prove (3.10). We note that the function $f\left(y+\varepsilon_{\perp}\right)$ can be written as

$$
f\left(y+\varepsilon_{\perp}\right)=\lim _{N \rightarrow \infty} \min _{\alpha\rangle \in \mathscr{H}}\left\langle\alpha\left|H\left(y+\varepsilon_{\perp}\right)\right| \alpha\right\rangle,
$$

for $y \in \mathscr{M}$, where $|\alpha\rangle$ shall be normalized as $\langle\alpha \mid \alpha\rangle=1$. We denote by $\mathscr{H}_{\mathrm{c}}$ the Hilbert space spanned by all the ground states in $\mathscr{M}$ :

$$
\mathscr{H}_{\mathrm{c}}=\operatorname{span}\left\{\left|0, y^{\prime}\right\rangle \mid y^{\prime} \in \mathscr{M}\right\} .
$$

The total Hilbert space is then decomposed as

$$
\mathscr{H}=\mathscr{H}_{\mathrm{c}} \oplus \tilde{\mathscr{H}} .
$$


By definition, $\tilde{\mathscr{H}}$ is the subspace on which expectation values of Hamiltonian are always nonvanishing. Any element $|\beta\rangle$ in $\mathscr{H}_{\mathrm{c}}$ can be expanded as

$$
|\beta\rangle=\sqrt{K_{N}} \int d^{D} y^{\prime} \beta\left(y^{\prime}\right)\left|0, y^{\prime}\right\rangle
$$

where $\beta\left(y^{\prime}\right)$ is a function which is non-vanishing only when $y^{\prime} \in \mathscr{M}$. A generic normalized vector $|\alpha\rangle$ can be decomposed as

$$
|\alpha\rangle=|\beta\rangle+|\tilde{\alpha}\rangle
$$

where $|\beta\rangle$ is given by $(\underline{3.18}$ ) and $|\tilde{\alpha}\rangle \in \tilde{\mathscr{H}}$. The normalization condition becomes

$$
\langle\beta \mid \beta\rangle+\langle\tilde{\alpha} \mid \tilde{\alpha}\rangle=1 .
$$

By substituting (3.19) into 3.15), we obtain

$$
\begin{array}{r}
f\left(y+\varepsilon_{\perp}\right)=\lim _{N \rightarrow \infty|\beta\rangle,|\tilde{\alpha}\rangle} \min _{+}\left[\left\langle\beta\left|H\left(y+\varepsilon_{\perp}\right)\right| \beta\right\rangle+\left\langle\tilde{\alpha}\left|H\left(y+\varepsilon_{\perp}\right)\right| \beta\right\rangle\right. \\
\left.+\left\langle\beta\left|H\left(y+\varepsilon_{\perp}\right)\right| \tilde{\alpha}\right\rangle+\left\langle\tilde{\alpha}\left|H\left(y+\varepsilon_{\perp}\right)\right| \tilde{\alpha}\right\rangle\right]
\end{array}
$$

Notice that for any $|\beta\rangle$ and $|\tilde{\alpha}\rangle$, the second and the third terms are vanishing in the large- $N$ limit because of (3.5) and the orthogonality between $|\beta\rangle$ and $|\tilde{\alpha}\rangle$. Here, let us consider the case where $\left|\varepsilon_{\perp}\right|$ is very small and put

$$
\langle\beta \mid \beta\rangle=c\left|\varepsilon_{\perp}\right|^{a},
$$

where $a$ and $c$ are non-negative constants. Then, the last term in (3.24) can be written as

$$
\left(1-c\left|\varepsilon_{\perp}\right|^{a}\right) \frac{\left\langle\tilde{\alpha}\left|H\left(y+\varepsilon_{\perp}\right)\right| \tilde{\alpha}\right\rangle}{\langle\tilde{\alpha} \mid \tilde{\alpha}\rangle}
$$

Note that the second factor is positive definite and is estimated as $\mathscr{O}\left(\left|\varepsilon_{\perp}\right|^{0}\right)^{2}$. Hence, (3.23) is $\mathscr{O}\left(\left|\varepsilon_{\perp}\right|^{0}\right)$ except the case with $a=0$ and $c=1$, where (3.23) is vanishing. Similarly, by using (3.11), the first term in (3.24) can be estimated as $\mathscr{O}\left(\left|\varepsilon_{\perp}\right|^{a+2}\right)$. Thus, we can see that the minimum in (3.24) is saturated by $a=0$ and $c=1$. (3.24) is now reduced to

$$
f\left(y+\varepsilon_{\perp}\right)=\lim _{N \rightarrow \infty} \min _{|\beta\rangle \in \mathscr{H}_{\mathrm{c}}}\left\langle\beta\left|H\left(y+\varepsilon_{\perp}\right)\right| \beta\right\rangle,
$$

where $\langle\beta \mid \beta\rangle=1$. By substituting (3.18) into (3.24) and using (3.11), we finally obtain

$$
f\left(y+\varepsilon_{\perp}\right)=\frac{1}{2} \int d^{D} y^{\prime}\left|\hat{\beta}\left(y^{\prime}\right)\right|^{2}\left(y^{\prime \mu}-y^{\mu}-\varepsilon_{\perp}^{\mu}\right)^{2} .
$$

Here, $\hat{\beta}\left(y^{\prime}\right)$ is the large- $N$ limit of the function which saturates the minimum in (3.24). It is vanishing on the outside of $\mathscr{M}$ and from the normalization condition, it satisfies $\int d^{D} y^{\prime}\left|\hat{\beta}\left(y^{\prime}\right)\right|^{2}=1$. Obviously, the right-hand side of (3.25) is minimized when $\hat{\beta}\left(y^{\prime}\right)$ localizes at $y$. Thus, we finally obtain (3.10).

\footnotetext{
${ }^{2}$ If this is proportional to a positive power of $\left|\varepsilon_{\perp}\right|$, it contradicts when $\left|\varepsilon_{\perp}\right| \rightarrow 0$ with the fact that expectation values of Hamiltonian in $\tilde{\mathscr{H}}$ is always nonvanishing.
} 


\section{Poisson structure}

Let us consider a $D \times D$ real antisymmetric matrix defined as ${ }^{3}$

$$
W^{\mu v}(y)=-i \lim _{N \rightarrow \infty} C_{N}\left\langle 0, y\left|\left[X^{\mu}, X^{v}\right]\right| 0, y\right\rangle .
$$

As we will show below, for $y \in \mathscr{M}$, (4.1) gives a Poisson tensor on $\mathscr{M}$ [6]. Namely, it is a tangent bivector on $\mathscr{M}$ satisfying

$$
P^{\mu}{ }_{v}(y) W^{v \rho}(y)=W^{\mu \rho}(y), \quad(y \in \mathscr{M})
$$

and the Poisson bracket on $\mathscr{M}$ defined by

$$
\{f, g\}=W^{\mu v}\left(\partial_{\mu} f\right)\left(\partial_{v} g\right)
$$

satisfies the Jacobi identity.

Firstly, we show that $W^{\mu v}(y)$ is a tangent bivector, namely, it satisfies 4.2). For this purpose, we use the following relation:

$$
\left\langle 0, y\left|\left[X^{\mu}, X^{v}\right]\right| 0, y\right\rangle\left\langle 0, y\left|X_{v}\right| n, y\right\rangle \sim\left(E_{n}(y)-E_{0}(y)\right)\left\langle 0, y\left|X^{\mu}\right| n, y\right\rangle,
$$

where $\sim$ stands for an equality for the leading-order terms in the large- $N$ limit. The relation (4.4) is proved as follows. By using (1.1) and (3.4), we obtain

$$
\left\langle 0, y\left|\left[X^{\mu}, X^{v}\right]\right| 0, y\right\rangle\left\langle 0, y\left|X_{v}\right| n, y\right\rangle \sim \frac{1}{2}\left\langle 0, y\left|\left\{X^{v}-y^{v},\left[X^{\mu}, X_{v}-y_{v}\right]\right\}\right| n, y\right\rangle .
$$

Then, note that $\left\{X^{v}-y^{v},\left[X^{\mu}, X_{v}-y_{v}\right]\right\}=2\left[X^{\mu}, H(y)\right]$. By substituting this into (4.5) we obtain (4.4). Now, let us compute

$$
P_{\rho}^{\mu}(y) W^{\rho v}(y)=\lim _{N \rightarrow \infty}\left[2 C_{N} \operatorname{Im}\left\{\sum_{n \neq 0} \frac{\left\langle 0, y\left|X^{\mu}\right| n, y\right\rangle\left\langle n, y\left|X^{\rho}\right| 0, y\right\rangle}{E_{n}(y)-E_{0}(y)}\left\langle 0, y\left|\left[X_{\rho}, X^{v}\right]\right| 0, y\right\rangle\right\}\right] .
$$

By using (4.4) and the completeness relation $\sum_{n=0}^{N-1}|n, y\rangle\langle n, y|=1$, we can calculate as

$$
P_{\rho}^{\mu}(y) W^{\rho v}(y)=\lim _{N \rightarrow \infty}\left[2 C_{N} \operatorname{Im}\left\{\left\langle 0, y\left|X^{\mu} X^{v}\right| 0, y\right\rangle-\left\langle 0, y\left|X^{\mu}\right| 0, y\right\rangle\left\langle 0, y\left|X^{v}\right| 0, y\right\rangle\right\}\right] .
$$

Note that the second term is zero since $X^{\mu}$ are Hermitian and the first term is equal to $W^{\mu v}(y)$. Thus, we obtain 4.2).

Secondly, we prove that the bracket defined in (4.3) satisfies the Jacobi identity. The Jacobi identity is equivalent to

$$
W^{\mu v}(y) \partial_{\mu} W^{\rho \sigma}(y)+W^{\mu \rho}(y) \partial_{\mu} W^{\sigma v}(y)+W^{\mu \sigma}(y) \partial_{\mu} W^{v \rho}(y)=0,
$$

for $y \in \mathscr{M}$. Let us consider an arbitrary polynomial $\Phi(x)$ with $D$ variables $x^{\mu}$, where the definition of the polynomial is $N$-independent. Because of [3.5), we have a relation

$$
\Phi(y)=\lim _{N \rightarrow \infty}\langle 0, y|\Phi(X)| 0, y\rangle .
$$

\footnotetext{
${ }^{3}$ Note that we use the same notation for this matrix and the polynomial which appears in the right-hand side of 1.1 . This is because the matrix 4.1) is indeed obtained by replacing $X^{\mu}$ with $y^{\mu}$ in the polynomial 1.1 (See 3.5).
} 
Then, as we will show below, the following relation holds:

$$
\lim _{N \rightarrow \infty}\left(-i C_{N}\right)\left\langle 0, y\left|\left[X^{\mu}, \Phi(X)\right]\right| 0, y\right\rangle=W^{\mu v}(y) \partial_{\nu} \Phi(y) .
$$

If we put $\Phi(X)=W^{\mu v}(X)=-i C_{N}\left[X^{\mu}, X^{v}\right]+\mathscr{O}\left(1 / C_{N}\right)$ in the above equation, (4.8) immediately follows from the Jacobi identity of the matrix commutators. So below, we will try to prove the relation 4.10).

The right-hand side of (4.10) is the large- $N$ limit of

$$
-i C_{N}\left\langle 0, y\left|\left[X^{\mu}, X^{v}\right]\right| 0, y\right\rangle \partial_{v}\langle 0, y|\Phi(X)| 0, y\rangle .
$$

The derivative of $|0, y\rangle$ is obtained from the formula in the perturbation theory as

$$
\partial_{\mu}|0, y\rangle=\sum_{n \neq 0} \frac{|n, y\rangle\left\langle n, y\left|X^{\mu}\right| 0, y\right\rangle}{E_{n}(y)-E_{0}(y)}+i A_{\mu}(y)|0, y\rangle,
$$

where $A_{\mu}(y)$ is a real number and correspond to the Berry connection of the ground states. By substituting this and using (4.4), we find that the large- $N$ limit of (4.11) is also equal to the lefthand side of 4.10). Thus, we obtain 4.10).

\section{Examples}

\subsection{Fuzzy sphere}

The fuzzy sphere is defined by

$$
X^{\mu}=\frac{2}{\sqrt{N^{2}-1}} L^{\mu}
$$

where $L^{\mu}$ are the $N$-dimensional irreducible representation matrices of the $S U(2)$ generators. The normalization factor is chosen so that $\left(X^{\mu}\right)^{2}=1$. The matrices satisfy the commutation relation,

$$
\left[X^{\mu}, X^{v}\right]=\frac{2 i}{\sqrt{N^{2}-1}} \varepsilon^{\mu v \rho} X_{\rho}
$$

The Hamiltonian is given by

$$
H(y)=\frac{1}{2}\left(1+|y|^{2}\right)-\frac{2}{\sqrt{N^{2}-1}} y^{\mu} L_{\mu},
$$

where $|y|=\sqrt{y_{\mu} y^{\mu}}$. As shown in appendix $\mathrm{A}$, in this case, the function $f(y)$ in (2.4) is given by

$$
f(y)=\frac{1}{2}(1-|y|)^{2} .
$$

So indeed the classical space (2.3) is $S^{2}$ with the unit radius.

From (3.1) and (4.1), we can easily derive explicit forms of the projection matrix and Poisson tensor. They are given as follows.

$$
\begin{aligned}
& P_{v}^{\mu}(y)=\frac{1}{|y|}\left(\delta_{v}^{\mu}-\frac{y^{\mu} y_{v}}{|y|^{2}}\right), \\
& W^{\mu v}(y)=2 \varepsilon^{\mu v \rho} \frac{y_{\rho}}{|y|},
\end{aligned}
$$

From these expressions, we can see that for $y \in \mathscr{M}$ (i.e. for $|y|=1$ ), they satisfy the required properties such as the projectivity for $P^{\mu}{ }_{v}$ and (4.2) and (4.8) for $W^{\mu v}$. 


\subsection{Fuzzy torus}

Next, we consider the fuzzy torus, which is defined by the two unitary matrices $U$ and $V$ satisfying the algebra

$$
V U=e^{i \theta} U V
$$

where $\theta=2 \pi / N$. The matrices $U$ and $V$ are explicitly given by the clock and shift matrices as

$$
\begin{aligned}
& U_{m n}=\delta_{m n} e^{i n \theta}, \\
& V_{m n}=\delta_{m+1 n},
\end{aligned}
$$

where $m, n=0,1, \cdots, N-1$ and $\delta_{m n}$ is the cyclic version of the Kronecker delta which satisfies $\delta_{N n} \equiv \delta_{0 n}$. We introduce four Hermitian matrices $X^{\mu}(\mu=1,2,3,4)$ as the real and imaginary parts of $U$ and $V$ as

$$
U=X^{1}+i X^{2}, \quad V=X^{3}+i X^{4}
$$

Then, the fuzzy torus can be regarded as the non-commutative Clifford torus embedded in the four dimensional flat space. Note that $X^{1}$ and $X^{2}$ commute with each other and satisfy $\left(X^{1}\right)^{2}+\left(X^{2}\right)^{2}=$ $\mathbf{1}_{N \times N}$ because of the unitarity condition of $U$, and so do $X^{3}$ and $X^{4}$.

The Hamiltonian (2.1) is given by

$$
H(y)=\frac{1}{2}(U-z)\left(U^{\dagger}-\bar{z}\right)+\frac{1}{2}(V-w)\left(V^{\dagger}-\bar{w}\right),
$$

where we introduced complex variables, $z=y^{1}+i y^{2}$ and $w=y^{3}+i y^{4}$. As shown in appendix B, the function $f(y)$ in (2.4) is given in this case by

$$
f(y)=\frac{1}{2}(1-|z|)^{2}+\frac{1}{2}(1-|w|)^{2}
$$

Hence, the classical space $\mathscr{M}$ is the Clifford torus given by $|z|=|w|=1$.

We can also obtain the projection operator from 3.1. We find

$$
P_{b}^{a}(y)=\frac{1}{|z|}\left(\delta_{b}^{a}-\frac{y^{a} y_{b}}{|z|^{2}}\right), \quad P_{\alpha}^{a}(y)=0, \quad P_{\beta}^{\alpha}(y)=\frac{1}{|w|}\left(\delta_{\beta}^{\alpha}-\frac{y^{\alpha} y_{\beta}}{|w|^{2}}\right),
$$

where $a, b=1,2$ and $\alpha, \beta,=3,4$. We can see that $P^{\mu}{ }_{v}$ is indeed a projection operator for $y \in \mathscr{M}$ and gives the standard induced metric of the Clifford torus.

In order to compute the Poisson tensor, we rewrite the algebra (5.6) using $X^{\mu}$ as

$$
\begin{aligned}
& {\left[X_{1} \pm i X_{2}, X_{3} \pm i X_{4}\right] \sim i \theta\left(X_{1} \pm i X_{2}\right)\left(X_{3} \pm i X_{4}\right),} \\
& {\left[X_{1} \pm i X_{2}, X_{3} \mp i X_{4}\right] \sim-i \theta\left(X_{1} \pm i X_{2}\right)\left(X_{3} \mp i X_{4}\right),}
\end{aligned}
$$

where we have neglected higher order terms in $\theta$. By putting $C_{N}=1 / \theta$ in 1.1 , we obtain the Poisson tensor on the Clifford torus as

$$
W^{\mu v}(y)=\left(\begin{array}{cccc}
0 & 0 & y^{2} y^{4} & -y^{2} y^{3} \\
0 & 0 & -y^{1} y^{4} & y^{1} y^{3} \\
-y^{2} y^{4} & y^{1} y^{4} & 0 & 0 \\
y^{2} y^{3} & -y^{1} y^{3} & 0 & 0
\end{array}\right) .
$$




\section{Summary}

In this paper, we considered the classical limit of matrix geometry described by a sequence of $D$ Hermitian matrices, $\left\{\left(X_{1}^{(N)}, \cdots, X_{D}^{(N)}\right) \mid N \in I\right\}$. We explained a prescription [6] to define the classical geometry for the matrix geometry. The classical space $\mathscr{M}$ is defined in terms of the Hamiltonian (2.1) and is given by the loci of zeros of the ground state energy in the large- $N$ limit as 2.3), while the tangent space of $\mathscr{M}$ is defined in terms of the projection matrix 3.1). We focused on the case where the matrices satisfy 1.1. We showed that in this case the classical space possesses a Poisson structure ${ }^{4}$. Under the assumption that the ground state energy is nondegenerate near $\mathscr{M}$, we found formulas (3.3) and (4.1) which relate the geometric objects to the matrix configurations.

\section{Acknowledgments}

This work was supported, in part, by Program to Disseminate Tenure Tracking System, MEXT, Japan.

\section{A. Classical geometry of fuzzy sphere}

In this appendix, we show that the function $f(y)$ for the fuzzy sphere is given by (5.4). Firstly, we consider a rotation matrix, which rotates a vector $v^{i}=(0,0,1)$ in $R^{3}$ to a general point $x^{i}$ on $S^{2}$ with a unit radius.

$$
x^{i}=\left(\Lambda^{-1}(x)\right)^{i}(x) v^{j} .
$$

Here, by using the standard poler coordinates $(\theta, \phi), x^{i}$ shall be expressed as

$$
x^{1}=\sin \theta \cos \phi, \quad x^{2}=\sin \theta \sin \phi, \quad x^{3}=\cos \theta .
$$

Note that the definition of $\Lambda$ has an ambiguity for the left action of $S O(2)$ rotations. We fix this ambiguity by setting

$$
\Lambda(x)=\left(\begin{array}{ccc}
\cos \phi & -\sin \phi & 0 \\
\sin \phi & \cos \phi & 0 \\
0 & 0 & 1
\end{array}\right)\left(\begin{array}{ccc}
\cos \theta \cos \phi & \cos \theta \sin \phi & -\sin \theta \\
-\sin \phi & \cos \phi & 0 \\
\sin \theta \cos \phi & \sin \theta \sin \phi & \cos \theta
\end{array}\right) .
$$

This definition is chosen in such a way that it becomes consistent with the definition of the Bloch coherent state in literatures introduced below. The matrix $\Lambda$ can also be written as the three dimensional representation matrix of the group element

$$
\hat{g}=e^{-i \hat{L}_{3} \phi} e^{i \hat{L}_{2} \theta} e^{i \hat{L}_{3} \phi},
$$

where $\hat{L}_{i}$ are the generators of the Lie algebra of $S U(2)$ satisfying $\left[\hat{L}_{i}, \hat{L}_{j}\right]=i \varepsilon_{i j k} \hat{L}_{k}$.

Second, let us consider an $N$ dimensional irreducible representation matrices $L_{i}=D_{N}\left[\hat{L}_{i}\right]$ of the $S U(2)$ generators. Note that any representation matrices of $S U(2)$ generators are invariant

\footnotetext{
${ }^{4}$ In this paper, we only considered the Poisson structure. However, recently, we also found that the classical space for the present setup possesses not only the Poisson structure but also a Kähler structure [14].
} 
tensors. This means that for any $S O(3)$ rotation of the vector index of the representation matrices, there exists a unitary similarity transformation which cancels the $S O(3)$ rotation. Hence, for the rotation made by $\Lambda(x)$, there exists a $N \times N$ unitary matrix $U(x)$ such that

$$
U^{\dagger}(x) L_{i} U(x)=L_{j}(\Lambda(x))^{j}{ }_{i}
$$

If we write $\Lambda(x)=D_{3}[\hat{g}], U(x)$ can be written as $U(x)=D_{N}[\hat{g}]$.

Now, let us diagonalize the Hamiltonian (5.3) for the fuzzy sphere. We write $y^{i}=|y| x^{i}$, where $x^{i}$ is the coordinates on the unit sphere given by A.2. Substituting (A.1) into this expression, we obtain $y^{i}=|y|\left(\Lambda^{-1}\right)^{i}{ }_{j} v^{j}$. Then by using this and (A.5), we can write the Hamiltonian (5.3) as

$$
H(y)=U(x)\left[\frac{1}{2}\left(1+|y|^{2}\right)-\frac{2|y|}{\sqrt{N^{2}-1}} L_{3}\right] U^{\dagger}(x) .
$$

Thus, the Hamiltonian is diagonalized by

$$
|n, y\rangle=U(x)|J J-n\rangle
$$

Here, we have adopted the standard notation $|J m\rangle(m=-J,-J+1, \cdots, J)$ for the basis vectors of the $N$-dimensional representation space, where $J$ is the spin of the representation given by $J=$ $(N-1) / 2$. The basis satisfies

$$
L_{3}|J m\rangle=m|J m\rangle, \quad L_{ \pm}|J m\rangle=\sqrt{(J \mp m)(J \pm m+1)}|J m \pm 1\rangle,
$$

where $L_{ \pm}=L_{1} \pm L_{2}$.

In particular, in A.7 , the ground state $|0, y\rangle=U(x)|J J\rangle$ is what is known as the Bloch coherent states. It satisfies

$$
H(y)|0, y\rangle=\left[\frac{1}{2}\left(1+|y|^{2}\right)-\frac{2 J|y|}{\sqrt{N^{2}-1}}\right]|0, y\rangle
$$

This leads to (5.4).

\section{B. Classical geometry of fuzzy torus}

In this appendix, we show that the function $f(y)$ for the Hamiltonian 5.9 is given by 5.10 . First, we note the following inequalities,

$$
\begin{aligned}
E_{0}(y) & =\min _{|\psi\rangle}\langle\psi|H(y)| \psi\rangle \\
& \geq \frac{1}{2} \min _{|\psi\rangle}\left\langle\psi\left|(U-z)\left(U^{\dagger}-\bar{z}\right)\right| \psi\right\rangle+\frac{1}{2} \min _{|\psi\rangle}\left\langle\psi\left|(V-w)\left(V^{\dagger}-\bar{w}\right)\right| \psi\right\rangle \\
& \geq \frac{1}{2}(1-|z|)^{2}+\frac{1}{2}(1-|w|)^{2} .
\end{aligned}
$$

The last inequality follows from the fact that the spectrum of any unitary matrix is contained in the unit circle on the complex plane. On the other hand, for any normalized state vector $|\alpha\rangle$, we have

$$
E_{0}(y) \leq\langle\alpha|H(y)| \alpha\rangle
$$


Hence, if there exists a normalized state vector $|\alpha\rangle$ which satisfies

$$
\lim _{N \rightarrow \infty}\langle\alpha|H(y)| \alpha\rangle=\frac{1}{2}(1-|z|)^{2}+\frac{1}{2}(1-|w|)^{2},
$$

the equation (5.10) immediately follows from (B.1) and (B.2). In the following, we construct such a state that satisfies $(B .3)$.

We introduce the basis used in equation (5.7),

$$
U|m\rangle=e^{i \theta}|m\rangle, \quad V|m\rangle=|m+1\rangle .
$$

For convenience, we extend the range of $m$ to a set of all integers by imposing the periodicity condition $|m+N\rangle=|m\rangle$. We then consider a state vector

$$
|\alpha\rangle:=\tilde{c} \sum_{m=-\left[\frac{N-1}{2}\right]}^{\left[\frac{N}{2}\right]} e^{-\frac{a}{2}\left(\frac{m}{N}-\frac{\arg z}{2 \pi}\right)^{2}} e^{-i(\arg w) m}|m\rangle,
$$

where $a$ is a constant of $\mathscr{O}(\sqrt{N}), \tilde{c}$ is a normalization constant determined by $\langle\alpha \mid \alpha\rangle=1$ and $[x]$ stands for the floor function defined by $[x]=\max \{m \in \mathbf{Z} \mid m \leq x\}$. In the large- $N$ limit, we can evaluate $\tilde{c}$ as

$$
\tilde{c}^{-2}=\sum_{m=-\left[\frac{N-1}{2}\right]}^{\left[\frac{N}{2}\right]} e^{-a\left(\frac{m}{N}-\frac{\arg z}{2 \pi}\right)^{2}} \sim N \int_{-\infty}^{\infty} d x e^{-a\left(x-\frac{\arg z}{2 \pi}\right)^{2}}=N \sqrt{\frac{\pi}{a}} .
$$

Here we approximated the discrete sum by the integral. By repeating almost same calculations, we can obtain

$$
\lim _{N \rightarrow \infty}\langle\alpha|U| \alpha\rangle=e^{i \arg z}, \quad \lim _{N \rightarrow \infty}\langle\alpha|V| \alpha\rangle=e^{i \arg w} .
$$

These relations imply that $|\alpha\rangle$ satisfies (B.3). Thus, we have proved (5.10).

\section{References}

[1] T. Banks, W. Fischler, S. H. Shenker and L. Susskind, Phys. Rev. D 55, 5112 (1997).

[2] N. Ishibashi, H. Kawai, Y. Kitazawa and A. Tsuchiya, Nucl. Phys. B 498, 467 (1997).

[3] D. Berenstein and E. Dzienkowski, Phys. Rev. D 86, 086001 (2012).

[4] A. Chatzistavrakidis, H. Steinacker and G. Zoupanos, JHEP 1109, 115 (2011).

[5] L. Schneiderbauer and H. C. Steinacker, "Measuring finite Quantum Geometries via Quasi-Coherent States," arXiv:1601.08007 [hep-th].

[6] G. Ishiki, Phys. Rev. D 92, no. 4, 046009 (2015).

[7] J. Arnlind, M. Bordemann, L. Hofer, J. Hoppe and H. Shimada, JHEP 0906, 047 (2009).

[8] J. Arnlind, J. Hoppe and G. Huisken, J. Diff. Geom. 91, 1 (2012).

[9] H. Grosse and P. Presnajder, Lett. Math. Phys. 28, 239 (1993). 
[10] H. Grosse and P. Presnajder, Lett. Math. Phys. 33, 171 (1995).

[11] A. B. Hammou, M. Lagraa and M. M. Sheikh-Jabbari, Phys. Rev. D 66, 025025 (2002).

[12] M. H. de Badyn, J. L. Karczmarek, P. Sabella-Garnier and K. H. C. Yeh, JHEP 1511, 089 (2015).

[13] J. L. Karczmarek and K. H. C. Yeh, JHEP 1511, 146 (2015).

[14] G. Ishiki, T. Matsumoto and H. Muraki, "Kähler structure in the commutative limit of matrix geometry," arXiv:1603.09146 [hep-th]. 